\title{
A NOTE ON THE MINIMUM MODULUS OF A CLASS OF INTEGRAL FUNCTIONS
}

\author{
S. M. SHAH
}

A well known theorem due to Littlewood, Wiman, and Valiron ${ }^{1}$ states that for any integral function of order less than one-half,

$$
\log m(r)>\text { (a positive constant) } \log M(r),
$$

on a sequence of circles of indefinitely increasing radius. I consider in this note a class of integral functions which have this property and prove the following theorem.

THEOREM 1. Hypothesis:

(1) $\left(R_{n}\right)$ is any sequence of positive numbers such that $R_{1}>1$, $R_{n} / R_{n-1} \geqq \lambda>1$.

(2) $\left(p_{n}\right)$ is any sequence of positive integers.

(3) $a_{11}, a_{12}, \cdots, a_{1_{1}}, a_{21}, \cdots, a_{2 p_{2}}, \cdots$ are a set of points such that $0<\left|a_{11}\right| \leqq\left|a_{12}\right| \leqq \cdots$ and such that a finite number $a_{n 1}, \cdots, a_{n} p_{n}$ lie inside the ring $\left(R_{n}-R_{n}^{\alpha}<|z|<R_{n}\right)$ where $0<\alpha<1$.

(4) $\mu_{n}$ is a sequence of positive integers such that $\sum_{1}^{\infty} p_{n} / \beta^{\mu_{n}}$ is convergent, $\beta$ being any constant greater than one.

(5) The exponent of convergence of the points

$$
a_{n r} \exp \left(2 \pi i \nu / \mu_{n}\right),
$$

where $r=1,2, \cdots, p_{n} ; \nu=0,1,2, \cdots, \mu_{n}-1 ; n=1,2,3, \cdots$, is $\rho$ $(0 \leqq \rho<\infty)$.

$(6)^{2}$ Lower bound $\left\{\mu_{n}\right\} \geqq 1+\rho$.

Conclusion:

(7) The canonical product

$$
f(z)=\prod_{n=1}^{\infty} \prod_{s=1}^{p_{n}}\left\{1-\frac{z^{\mu_{n}}}{a_{n s}^{\mu_{n}}}\right\}
$$

formed with these points as zeros is of order $\rho$; and the values of $r=|z|$ for which the inequality

$$
m(r, f)>C M(r, f),
$$

Received by the editors February 4, 1946, and, in revised form, November 29, 1946.

${ }^{1} \mathrm{G}$. Valiron, Lectures on the general theory of integral functions, pp. 128-130.

${ }^{2}$ It is possible to choose $R_{n}, p_{n}$, and so on, satisfying the conditions (1) to (6). Example: $R_{n}=2^{2 n} ; p_{n}=n^{22^{n}} ; \mu_{n}=2^{n}$. Here $\rho=1$. 
where $C=C(\lambda, \epsilon)>0$, is satisfied form a set of upper density greater than $1-1 / \lambda-\epsilon$.

THEOREM 2. If (1), (2), (3), (4), (5), and (6) hold and if $\rho>0$ and if further ${ }^{2}$

$$
\sum_{n=1}^{N} \mu_{n} p_{n} / R_{N}^{\rho} \rightarrow \infty \quad \text { with } N \rightarrow \infty
$$

then

$$
\limsup _{r \rightarrow \infty} \log m(r, f) / r^{\rho}=\infty
$$

where $f$ is the canonical product (8); and the values of $r$ for which $\log m(r, f)>\Delta r^{\rho}$ where $\Delta$ is any arbitrarily large constant form a set of upper density greater than $1-1 / \lambda-\epsilon$.

THEOREM 3. Hypothesis: Let $\rho>0$ be nonintegral and (1), (2), (3), (4), and (5) hold. ${ }^{3}$

Conclusion:

(10) Any integral function of order $\rho$ with exactly these zeros will be of the form

$$
F(z)=e^{o(z)} P(z) \prod_{n=n_{1}}^{\infty} \prod_{s=1}^{p_{n}}\left\{1-\frac{z^{\mu_{n}}}{a_{n s}^{\mu_{n}}}\right\},
$$

where $g(z)$ is a polynomial of degree not exceeding $\rho, P(z)$ a polynomial ; and the values of 4 for which

$$
\log m(r, F)>(1-\epsilon) \log M(r, F)
$$

holds will form a set of upper density greater than $1-1 / \lambda-\epsilon$.

ThEOREM 4. If $\rho>0$ and (1), (2), (3), (4), (5), and (9) hold then conclusion (10) holds.

THEOREM 5. If (1), (2), (3), (4), (5), and (6) hold and if $m_{\sigma}(r)$ and $M_{\sigma}(r)$ denote the lower and upper bounds of $|f(z)|$, where $f(z)$ is the canonical product (8), of order $\rho(0 \leqq \rho<\infty)$ in the annulus $r \leqq|z|$ $\leqq r+r^{\sigma}(\sigma<1-\rho)$ then the values of $r$ for which ${ }^{6}$

${ }^{8}$ For instance $R_{n}=2^{2 n}, p_{n}=n ; \mu_{n}=2^{7(n-1)}$. Here $\rho=7 / 2$.

' $P(z)$ is a polynomial having zeros at points $a_{n r} \exp \left(2 \pi i v / \mu_{n}\right), r=1,2, \cdots, p_{n}$; $\nu=0,1,2, \cdots, \mu_{n}-1$ and $n=1,2, \cdots, n_{1}-1$ only.

5 See footnotes 2 and 3.

- For a number of results on the flat regions of integral functions, see J. M. Whittaker, $A$ property of integral functions of finite order, Quart. J. Math. Oxford Ser. vol. 2 (1931) pp. 252-258; B. J. Maitland, The flat regions of integral functions of finite order, ibid. vol. 15 (1944) pp. 84-96; and the references mentioned in the paper of Maitland. 


$$
m_{\sigma}(r)>C_{1} M_{\sigma}(r),
$$

where $C_{1}=C_{1}(\lambda, \epsilon)>0$, holds form a set of upper density greater than $1-1 / \lambda-\epsilon$.

Proof of Theorem 1. Let $|z|=R=\lambda^{\gamma} R_{k}(0<\gamma<1)$, where $k$ is so large that

$$
\lambda^{\gamma} R_{k}<R_{k+1}-R_{+1}^{\alpha}
$$

$f(z)=P_{1} P_{2}$, where

$$
\begin{aligned}
P_{1} & =\prod_{n=1}^{k} \prod_{s=1}^{p_{n}}\left\{1-\frac{z^{\mu_{n}}}{a_{n s}^{\mu_{n}}}\right\} \\
P_{2} & =\prod_{n=k+1}^{\infty} \prod_{s=1}^{p_{n}}\left\{1-\frac{z^{\mu_{n}}}{a_{n s}^{\mu_{n}}}\right\}, \\
\left|P_{1}\right| & \leqq \prod_{n=1}^{k} \prod_{s=1}^{p_{n}}\left\{1+\frac{R^{\mu_{n}}}{\left|a_{n s}\right|^{\mu_{n}}}\right\} \\
& =\left(\prod_{n=1}^{k} \prod_{s=1}^{p_{n}} \frac{R^{\mu_{n}}}{\left|a_{n s}\right|^{\mu_{n}}}\right)\left(\prod_{n=1}^{k} \prod_{s=1}^{p_{n}}\left\{1+\frac{\left|a_{n s}\right|^{\mu_{n}}}{R^{u_{n}}}\right\}\right) \\
& =P_{11} P_{12},
\end{aligned}
$$

say. Now $\left|a_{n s}\right|<R_{n}$,

$$
\left|P_{12}\right| \leqq \prod_{1}^{k}\left\{1+\left(\frac{R_{n}}{R}\right)^{\mu_{n}}\right\}^{p_{n}},
$$

and $R_{n} / R \leqq 1 / \lambda^{\gamma}<1$ for $n=1,2, \cdots, k$, and $\sum p_{n} / \lambda^{\gamma \mu_{n}}$ is convergent. Hence

$$
\begin{aligned}
& \left|P_{12}\right| \leqq C_{2}, \\
& \left|P_{2}\right| \leqq \prod_{n=k+1}^{\infty} \prod_{s=1}^{p_{n}}\left\{1+\frac{R^{\mu_{n}}}{\left|a_{n s}\right|^{\mu_{n}}}\right\},
\end{aligned}
$$

where $\left|a_{n s}\right| \geqq\left|a_{k+1, s}\right| \geqq R_{k+1}-R_{k+1}^{\alpha}$,

$$
\frac{R}{\left|a_{n s}\right|} \leqq \frac{R}{R_{k+1}-R_{k+1}^{\alpha}} \sim \frac{\lambda^{\gamma} R_{k}}{R_{k+1}} \leqq \frac{1}{\lambda^{1-\gamma}},
$$

and $\sum p_{n} / \lambda^{(1-\gamma) \mu_{n}}$ is convergent. Hence

$$
\left|P_{2}\right| \leqq C_{3}
$$

${ }^{7} C, C_{1}, C_{2}, \cdots$ denote finite positive (nonzero) constants. 
and so

Further

$$
M(R) \leqq C_{2} C_{3} \prod_{n=1}^{k} \prod_{s=1}^{p_{n}} \frac{R^{\mu_{n}}}{\left|a_{n s}\right|^{\mu_{n}}}
$$

$$
\begin{aligned}
\left|P_{1}\right| & =\prod_{n=1}^{k} \prod_{s=1}^{p_{n}}\left|1-\frac{z^{\mu_{n}}}{a_{n s}^{\mu_{n}}}\right| \\
& \geqq \prod_{n=1}^{k} \prod_{s=1}^{p_{n}}\left\{\frac{R^{\mu_{n}}}{\left|a_{n s}\right|^{\mu_{n}}}-1\right\} \\
& \geqq\left(\prod_{n=1}^{k} \prod_{s=1}^{p_{n}} \frac{R^{\mu_{n}}}{\left|a_{n s}\right|^{\mu_{n}}}\right)\left(\prod_{n=1}^{k} \prod_{s=1}^{p_{n}}\left\{1-\frac{\left|a_{n s}\right|^{\mu_{n}}}{R^{\mu_{n}}}\right\}\right) \\
& =P_{11} P_{14}
\end{aligned}
$$

say. Since $\sum p_{n} / \lambda^{\gamma \mu_{n}}$ is convergent and

$$
\begin{aligned}
& \left|P_{2}\right| \geqq \prod_{n=k+1}^{\infty} \prod_{s=1}^{p_{n}}\left\{1-\frac{R^{\mu_{n}}}{\left|a_{n s}\right|^{\mu_{n}}}\right\} \geqq C_{5}, \\
& m(R) \geqq C_{4} C_{5} \prod_{n=1}^{k} \prod_{s=1}^{p_{n}} \frac{R^{\mu_{n}}}{\left|a_{n s}\right|^{\mu_{n}}} \cdots,
\end{aligned}
$$

which gives that $m(R) \geqq C_{6} M(R)$ where $C_{6}=C_{6}(\lambda, \gamma)$. Now given $\epsilon>0$ let $\epsilon_{1}=\epsilon \lambda^{2} /(\lambda+1+\epsilon \lambda)$. Writing $\lambda \gamma=\theta$ and $R=\theta R_{k}$, where $1+\epsilon_{1} \leqq \theta$ $\leqq \lambda-\epsilon_{1}$ and $k \geqq K, K$ being so large that $R_{K}\left(\lambda-\epsilon_{1}\right)<R_{K+1}-R_{K+1}^{\alpha}$, we get $m(R) \geqq C(\lambda, \epsilon) M(R)$. This inequality holds good over a set of upper density greater than

$$
\frac{\left(\lambda-\epsilon_{1}\right)-\left(1+\epsilon_{1}\right)}{\lambda-\epsilon_{1}}=1-\frac{1}{\lambda}-\epsilon .
$$

Proof of Theorem 2. We know from (12) that $m(R, f) \geqq C_{4} C_{5} X$, where

$$
X=\prod_{n=1}^{k} \prod_{s=1}^{p_{n}} \frac{R^{\mu_{n}}}{\left|a_{n s}\right|^{\mu_{n}}} \geqq \lambda^{\left(\gamma \Sigma_{1}^{k_{\mu_{n}} p_{n}}\right)}
$$

$\log m(R, f) \geqq \log \left(C_{4} C_{5}\right)+\log X \geqq \log \left(C_{4} C_{5}\right)+\gamma \log \lambda\left(\sum_{1}^{k} \mu_{n} p_{n}\right)$

$>\Delta R^{p}$

for all large $R$.

Hence $\lim \sup _{r \rightarrow \infty} \log m(r, f) / r^{\rho}=\infty$. Further, the values of $r$ for which $\log m(r, f)>\Delta r^{\rho}$ form a set of upper density greater than $1-1 / \lambda-\epsilon$. 
Proof of Theorem 3. Given $\epsilon>0$, let $\epsilon_{2}=\epsilon /(2-\epsilon)$. Since

$$
\sum \mu_{n} p_{n} /\left(R_{n}-R_{n}^{\alpha}\right)^{\rho-\epsilon_{8}}
$$

is divergent we have

$$
\mu_{n} p_{n} \geqq R_{n}^{\rho-\epsilon_{4}} \quad \text { or } n=k_{1}, k_{2} .
$$

Let $|z|=R=\lambda^{\gamma} R_{k}\left(0<\gamma<1\right.$ and $\left.1+\epsilon_{1} \leqq \lambda^{\gamma} \leqq \lambda-\epsilon_{1}\right)$, where $k$ takes the values $k_{1}, k_{2}, \cdots$. If $X=\prod_{n=n_{1}}^{k} \prod_{s=1}^{p_{n}} R^{\mu_{n}} /\left|a_{n s}\right|^{\mu_{n}}$ then $X \geqq \exp \left\{\gamma \log \lambda \sum_{n_{1}}^{k} \mu_{n} p_{n}\right\}$ and so $\log X \geqq C_{6} \sum_{n_{1}}^{\mathbf{k}} \mu_{n} p_{n} \geqq C_{6} R_{\mathbf{k}}^{\rho-e_{4}}=C_{7} R^{p-e_{4}}$. Choosing $k$ and hence $R$ sufficiently large we have, as in Theorem 1,

$$
\begin{aligned}
m(R, F) & >C_{8} \exp \left\{\log X-C_{9} R^{[\rho]}\right\}, \\
\log m(R, F) & >\log C_{8}+\log X-C_{9} R^{[\rho]} \\
& >\left(1-\epsilon_{2}\right) \log X .
\end{aligned}
$$

Similarly $\log M(R, F)<\left(1+\epsilon_{2}\right) \log X$ which gives

$$
\frac{\log m(R, F)}{\log M(R, F)}>\frac{1-\epsilon_{2}}{1+\epsilon_{2}}=1-\epsilon .
$$

As in Theorem 1, this result holds for values of $R$ forming a set of upper density greater than $1-1 / \lambda-\epsilon$.

Theorem 4 can be similarly proved.

Proof of Theorem 5. We know that for $|z|=R=\lambda^{\gamma} R_{k}(0<\gamma<1$, $\left.1+\epsilon_{1} \leqq \lambda^{\gamma} \leqq \lambda-\epsilon_{1}\right)$

$$
m(R, f) \geqq C_{4} C_{5} \prod_{n=1}^{k} \prod_{s=1}^{p_{n}} \frac{R^{\mu_{n}}}{\left|a_{n s}\right|^{\mu_{n}}} .
$$

We can choose $k$ so large that $R^{\prime}=R+R^{\sigma}<\lambda^{\gamma+{ }^{6}} R_{k}$, where $\gamma+\epsilon_{5}<1$,

$$
R^{\prime}<R_{k+1}-R_{k+1}^{\alpha}
$$

Now

$$
M\left(R^{\prime}, f\right)<C_{10} \prod_{n=1}^{k} \prod_{s=1}^{p_{n}} \frac{R^{\prime \mu_{n}}}{\left|a_{n s}\right|^{\mu_{n}}}
$$

and therefore

$$
\frac{m(R, f)}{M\left(R^{\prime}, f\right)}>\frac{C_{4} C_{5}}{C_{10}}\left(\frac{R}{R^{\prime}}\right)^{\Sigma_{n=1}^{h} \mu_{n} p_{n}} .
$$

Now $Y=\left(R^{\prime} / R\right)^{-\Sigma_{1}^{k} \mu_{n} p_{n}}=\left(1+R^{\sigma-1}\right)^{-\Sigma_{1}^{k} \mu_{n} p_{n}}$. Further $\sum_{1}^{k} \mu_{n} p_{n}$ $<\left(C_{11} \log R\right) R^{p+\epsilon 6}<R^{p+e r}$ for all large $R$. Hence $Y>\exp \left\{-R^{\rho+\epsilon r}\right.$ 
$\left.\log \left(1+R^{\sigma-1}\right)\right\}$ and $R^{\rho+\epsilon} \log \left(1+R^{\sigma-1}\right) \sim R^{\rho+\epsilon \gamma+\sigma-1} \rightarrow 0$ as $R \rightarrow \infty$, since $\sigma<1-\rho$ and $\epsilon_{7}$ can be chosen so small that $\sigma<1-\rho-\epsilon_{7}$. Hence $Y>1 / 2$ for all large $R$ and so

$$
\frac{m(R, f)}{M\left(R^{\prime}, f\right)}>\frac{C_{4} C_{5}}{2 C_{10}}
$$

Further

$$
\frac{m\left(R^{\prime}, f\right)}{M\left(R^{\prime}, f\right)}>C_{11}
$$

Hence

$$
\frac{m_{\sigma}(R)}{M_{\sigma}(R)}=\min \left\{\frac{m(R)}{M\left(R^{\prime}\right)}, \frac{m\left(R^{\prime}\right)}{M\left(R^{\prime}\right)}\right\} \geqq \min \left\{\frac{C_{4} C_{5}}{2 C_{10}}, C_{11}\right\} \geqq C_{1} .
$$

The values of $R$ for which this result holds form a set of upper density greater than $1-1 / \lambda-\epsilon$.

Added in proof. The positive numbers $\epsilon$ and $\epsilon_{4}$ are chosen so small that

$$
1 / \lambda+\epsilon<1 ; \quad[\rho]+\epsilon_{4}<\rho .
$$

In the proof of Theorem 1 we showed that

$$
M(R) \leqq C_{2} C_{3} P_{11} ; \quad m(R) \geqq C_{4} C_{5} P_{11},
$$

both relations holding for all $R$ such that

$$
\left(1+\epsilon_{1}\right) R_{k} \leqq R \leqq\left(\lambda-\epsilon_{1}\right) R_{k} \quad(k>K) .
$$

Here

$$
\begin{aligned}
& C_{2}=\prod_{n=1}^{\infty}\left\{1+\left(\frac{1}{1+\epsilon_{1}}\right)^{\mu_{n}}\right\}^{p_{n}}, C_{4}=\prod_{n=1}^{\infty}\left\{1-\left(\frac{1}{1+\epsilon_{1}}\right)^{\mu_{n}}\right\}^{p_{n}}, \\
& C_{8}=\prod_{n=1}^{\infty}\left\{1+\left(1-\frac{\epsilon_{1}}{2 \lambda}\right)^{\mu_{n}}\right\}^{p_{n}}, C_{5}=\prod_{n=1}^{\infty}\left\{1-\left(1-\frac{\epsilon_{1}}{2 \lambda}\right)^{\mu_{n}}\right\}^{p_{n}} .
\end{aligned}
$$

If $C=C_{4} C_{5} / C_{2} C_{3}$ we have

$$
m(R) \geqq C M(R),
$$

the inequality holding over a set of upper density greater than $1-1 / \lambda-\epsilon$. If we further suppose that $\lambda=R_{n} / R_{n-1}(n=2,3, \cdots)$, then this inequality holds good over a set of upper density greater than $1-\lambda \epsilon(1+\epsilon) /(\lambda-1)$.

MusLim University 